\title{
EDITORIAL
}

\section{Rejet zéro, niveau zéro, risque acceptable}

L'intérêt de développer et de disposer de méthodes d'élimination des polluants dans les eaux de distribution est évident. L'accident de contamination est une éventualité avec laquelie il faut toujours compter et il convient d'v faire face avec efficacité.

Amplifiés par les «tambours de bronze» que sont les médias, la pathologie du travail, la pollution de l'environnement, l'emploi d'additifs alimentaires, la mise en évidence de produits cancérigènes ou toxiques, l'exposition aux rayonnements ionisants ou non ionisants engendrent chez la population un sentiment diffus d'inquiétude. Certains groupes de pression s'appuient sur cette crainte pour réclamer un risque nul, une contamination nulle, une irradiation nulle..., d'où la doctrine du «rejet zéro».

Les milieux scientifiques et les responsables techniques en arrivent à être consternés surtout quand il s'agit de définir le «zéro» (1). Selon le "Larousse», le zéro se définit comme une «absence de valeur» ou encore comme le «point de départ de l'échelle de graduation d'un instrument de mesure», ce qui suppose un système de mesure convenablement adapté. Actuellement, le développement de méthodes analytiques affinées, la mise en œuvre de moyens sophistiqués permettent de retrouver un produit chimique alors qu'il y a quelques années, ce dernier n'était pas décelable. Il est vrai qu'à force d'abaisser les seuils de mesure, «tout se retrouvera dans tout». De ce fait, l'interprétation du «zéro à caractère évolutif» requerra de plus en plus de prudence. Par ailleurs, il ne faudrait pas que par un souci de perfectionnisme, on soit conduit à mettre en cuvre des technologies coûteuses sans que la nécessité ne s'en fasse sentir du point de vue sanitaire. De même, il ne conviendrait pas que I'on en vienne, par des habitudes de routine, à poursuivre indéfiniment des examens inutiles dont le caractère onéreux n'échappe à personne. L'anaIyse pour l'analyse ne peut constituer une doctrine de surveillance. Certes, les responsables de la réglementation en matière de contrôle de la pollution sont généralement raisonnables mais certains d'entre eux ressentent une espèce de démangeaison à imiter les législations étrangères les plus restrictives. La tentation pourrait alors être grande d'accroître d'une façon exponentielle les exigences administratives.

L'obtention d'un «niveau zéro» pour les normes de potabilité de l'eau souffre des mêmes difficultés. Il est bien évident que la détermination de la «concentration limite autorisée» (C.L.A.) doit tenir compte de l'apport alimentaire et d'autres apports éventuels, d'où la nécessité d'établir une limite d'ingestion dans le temps

(1) Etymologie du mot "zéro" (Littré) : zéro est une al tération de zefiro (italien), zefiro venant de l'arabe cifron, nom signifiant vide et zéro et traduisant ainsi exactement le sanscrit "çùnya" qui a les deux mêmes sens. Zéro et chiffre sont le même mot ; il est curieux de constater ainsi deux formes se différenciant pour signifier des choses très différentes. 
(hebdomadaire, mensuelle ou annuelle) correspondant aux différents vecteurs alimentaires. En cas de pollution accidentelle par des constituants chimiques, une exposition à des niveaux plus élevés peut éventuellement être tolérée en tenant compte des caractéristiques spécifiques de la substance en cause, de sa concentration et de la durée de la consommation. C'est alors aux autorités sanitaires de fixer un «niveau d'intervention» pour la mise en œuvre des moyens d'épuration. Cette attitude est plus satisfaisante que la doctrine peu réaliste du «niveau zéro», qui peut constituer une entrave au développement des activités humaines et, de par l'impossibilité de son application pratique, risque d'ouvrir la porte, par réaction excessive, à l'abus des facilités offertes par une doctrine laxiste.

La société moderne développe un certain nombre de risques parmi lesquels - il convient de distinguer le risque principal en évitant de s'égarer sur des risques secondaires d'où la nécessité d'une évaluation qualitative et quantitative des risques. Les activités de la vie humaine se déroulent au milieu de risques de toutes espèces : accidents du travail, accidents de circulation, accidents liés à la pratique de sports, accidents domestiques, etc... Certaines de ces activités ont un caractère indispensable, d'autres sont utiles à la vie quotidienne, d'autres sont simplement désirables, agréables ou intéressantes à divers points de vue. Ces risques de toutes espèces qui ne peuvent être supprimés sont, par là même, «acceptés». L'acceptation du risque par la population dépend du bénéfice qu'elle peut en retirer ; encore faut-il distinguer le risque que l'on accepte pour soi, du risque que l'on accepte pour les autres avec plus ou moins d'égoisme. Ainsi, l'automobiliste qui se promène accepte un risque d'accident pour lui-même alors que le chauffeur de car accepte professionnellement un risque pour les autres. Pour accepter le risque, il faut d'abord le rendre acceptable en le limitant à un certain niveau. C'est sur ce niveau acceptable et non sur la possibilité de supprimer le risque qu'il doit y avoir discussion. Pour certains, le terme de «risque acceptable» peut être difficile à admettre. Cette réticence peut se comprendre et il est possible de penser que l'on ne devrait pas accepter délibérément que certaines personnes puissent être exposées à des risques. Cependant, la notion de risque acceptable tient compte du fait tangible que l'individu ne peut objectivement renoncer à un certain nombre d'activités comportant des risques plus ou moins grands ; un équilibre est à rechercher entre un niveau de risque acceptable pour l'individu et la société, et les avantages qu'ils peuvent en retirer. Pratiquement, il n'est pas possible de garantir le développement d'une société sans risque, d'ailleurs une société sans risque serait-elle vraiment la meilleure des sociétés?

Au fil des années, nous avons appris à contrôler les crues des fleuves avec les barrages mais le risque de rupture d'un barrage n'est pas égal à zéro; nous avons appris à contrôler, le feu pour cuire nos aliments et nous chauffer mais le risque d'incendie n'est pas nul. En fait, un contrôle des risques a été pratiqué dans de nombreux cas, mais en présence des forces de la nature, le risque ne peut être totalement éliminé. II est assez paradoxal de constater que nous avons accepté la production de l'électricité et ses risques intrinsèques à partir de l'eau, du charbon, du fuel mais nous considérons comme inacceptable tout accident lié à l'énergie nucléaire.

En définitive, la fixation du niveau acceptable d'un risque sanitaire s'apparente à une recherche du type "coût-avantage». En dehors du fait qu'il y a des cas où la prévention du risque est impossible pratiquement, techniquement ou financièrement, il est bien évident que certaines interventions doivent avoir un caractère 
prioritaire et qu'ainsi d'autres seront obligatoirement retardées. En fait, l'analyse des risques consiste plutôt à définir des priorités que des principes.

Dans des situations particulières, il conviendrait de s'inspirer du principe établi par la Commission Internationale de Protection Radiologique et communément appelé le principe de "I'alara" («as low as reasonably achievable») qui fait apparaître le terme «raisonnable». II en découle que les dispositions de protection à appliquer doivent être en relation directe avec le risque présenté. Ces dispositions doivent avoir un caractère homogène grâce à une juste évaluation du risque qui est la condition première pour une appréciation valable des facteurs économiques et sociaux. D'ailleurs, l'Organisation Mondiale de la Santé reconnaît clairement la nécessité d'apprécier qualitativement et quantitativement le risque et le bénéfice sans négliger d'autres facteurs comme les possibilités techniques et économiques. 